\title{
Automatic Method for Identifying Photospheric Bright Points and Granules Observed by Sunrise
}

\author{
M. Javaherian ${ }^{1}$ - H. Safari ${ }^{1} \cdot$ A. Amiri ${ }^{2}$ \\ S. Ziaei $^{1}$
}

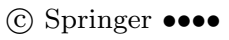

\begin{abstract}
In this study, we propose methods for the automatic detection of photospheric features (bright points and granules) from ultra-violet (UV) radiation, using a feature-based classifier. The methods use quiet-Sun observations at 214 $\mathrm{nm}$ and $525 \mathrm{~nm}$ images taken by Sunrise on 9 June 2009. The function of region growing and mean shift procedure are applied to segment the bright points (BPs) and granules, respectively. Zernike moments of each region are computed. The Zernike moments of BPs, granules, and other features are distinctive enough to be separated using a support vector machine (SVM) classifier.

The size distribution of BPs can be fitted with a power-law slope -1.5. The peak value of granule sizes is found to be about $0.5 \operatorname{arcsec}^{2}$. The mean value of the filling factor of BPs is 0.01 , and for granules it is 0.51 . There is a critical scale for granules so that small granules with sizes smaller than $2.5 \operatorname{arcsec}^{2}$ cover a wide range of brightness, while the brightness of large granules approaches unity. The mean value of $\mathrm{BP}$ brightness fluctuations is estimated to be 1.2, while for granules it is 0.22 . Mean values of the horizontal velocities of an individual BP and an individual BP within the network were found to be $1.6 \mathrm{~km} \mathrm{~s}^{-1}$ and 0.9 $\mathrm{km} \mathrm{s}^{-1}$, respectively. We conclude that the effect of individual BPs in releasing energy to the photosphere and maybe the upper layers is stronger than what the individual BPs release into the network.
\end{abstract}

Keywords: Sun: photosphere - Sun: Bright points - Sun: magnetic fields - Sun: granulation - Techniques: image processing

\section{Introduction}

The solar surface is stochastically covered by small-scale features such as bright points (BPs) and granules (Sánchez Almeida et al., 2004; de Wijn et al., 2008; Riethmüller et al., 2010) at times of both peak and minimum of the solar cycles

\footnotetext{
1 Department of Physics, University of Zanjan, University Blvd., 45371-38791, Zanjan, I. R. Iran, email: safari@znu.ac.ir

2 Department of Computer Engineering, University of

Zanjan, University Blvd., 45371-38791, Zanjan, I. R. Iran
} 
(Berger et al., 1995; Zhang et al., 1998). Statistical properties of these features are important subjects of solar physics.

BPs, first reported by Dunn and Zirker (1973), are linked to the emergence of small magnetic elements in the solar surface (Riethmüller et al., 2010). Statistically speaking, they cover approximately five percent of the solar surface in TiO $705.68 \mathrm{~nm}$ images (Andic et al., 2011) and are well observed in the G band and 214-515 nm wavelengths (de Wijn et al., 2008; Utz et al., 2009; Riethmüller et al., 2010).

Following Berger et al. (1995), visible-light signatures of photospheric magnetic concentrations have been classified into four main groups: faculae, filigree, facular BPs, and network BPs.

BPs highlight the emergence and evolution of magnetic structures on the solar surface and granulation pattern (Frazier and Stenflo, 1978; de Wijn et al., 2008; Nordlund, Stein, and Asplund, 2009).

Solar granulation is widely considered to be resulting from a convective turbulent process (Cloutman, 1979; Roudier and Muller, 1987). The granule characteristic scale is $\sim 1.35 \operatorname{arcsec}^{2}$, ranging widely from 0.3 to $4 \operatorname{arcsec}^{2}$ (Rusu, 2005; Robitaille, 2011). The average center-to-center granule spacing is about 1300 $\mathrm{km}$ with a wide range. A granular cell has a lifetime of about 8-15 minutes, and the typical vertical and horizontal velocities are approximately $1 \mathrm{~km} \mathrm{~s}^{-1}$ and $2 \mathrm{~km} \mathrm{~s}^{-1}$, respectively (Rusu, 2005). An extended review on solar surface magneto-convection is given by Stein (2012).

Solar space missions (e.g., TRACE, Hinode, and Sunrise) and ground-based telescopes for high-resolution solar observations (e.g., SST, NST, and GREGOR) provide images of the solar surface using various filters and cadences. To optimize the statistical analysis of various kinds of solar phenomena, it is necessary to develop automatic detection techniques. One of the significant methods for granules segmentation and finding BPs is multiple-level tracking (MLT4) based on pattern recognition methods (Bovelet and Wiehr, 2007). Kobel et al. (2010) employed another classification method to derive distinguishing properties of both BPs and faculae. Aschwanden (2010) has given an extensive overview of image processing-techniques and feature-recognition algorithms in solar data.

In the present study, we propose an automatic detection technique for BPs and granules at 214 and $525.02 \mathrm{~nm}$ in Sunrise images, taken of the quiet Sun, using Zernike moments, region growing, mean shift segmentation, and SVM.

The paper is organized as follows: first, the data reduction is discussed in Section 2. Identification methods of BPs and granules by using region growing, extracting Zernike moments, SVM, and applying the mean shift procedure are explained in Section 3. The results are given in Section 4. Finally, the conclusions are presented in Section 5.

\section{Data Analysis}

The Sunrise balloon-borne solar observatory was launched on 8 June 2009 (Barthol et al., 2011; Solanki et al., 2010). After reaching the required height in the Earth's atmosphere, the high-resolution images in the UV began to 
be recorded (Solanki et al., 2010). Sunrise has a resolution of about $100 \mathrm{~km}$. This observatory is equipped with the Sunrise filter imager (Gandorfer et al., 2011), an imaging magnetograph experiment (Martínez Pillet et al., 2011), an image stabilization and light distribution unit, and a correlating wavefront sensor (Berkefeld et al., 2011). The Sunrise Filter Imager (SuFI) is a diffraction-limited filter imager whose focal effective length is $121 \mathrm{~m}$ and which works in five different wavelength bands. The Imaging Magnetograph eXperiment (IMaX) data produce $936 \times 936$ pixels with a resolution of $0.15-0.18$ arcsec for studying solar magnetic and velocity fields. IMaX uses a Zeeman triplet with a Landé factor $\mathrm{g}=3$ (in the FeI $525.02 \mathrm{~nm}$ line). The Sunrise together with its instruments provides four levels of data. Level-0 are raw data, level-1 data are fully reduced, including phase-diversity reconstruction using individual and averaged wavefront exerts on both level-2 and level-3 data, respectively. We used a sequence of 32 images (level-2 data) recorded on 9 June 2009 (14:00-15:00 UT) at $214 \mathrm{~nm}$ (Figure 1A) and reconstructed by deconvolution using a modified Wiener filter and the pointspread function of the optical system that was derived from through calibration applying phase diversity (Solanki et al., 2010). The images were recorded using an array of $711 \times 1972$ pixels, with a pixel size of $0.02 \operatorname{arcsec}$ (both $x$ and $y$ directions). The exposure time and cadence at $214 \mathrm{~nm}$ were $30 \mathrm{~s}$ and $42 \mathrm{~s}$, respectively. In these images, both BPs and granulation were clearly observable (Riethmüller et al., 2010). Since intergranular lanes were not clearly visible at $214 \mathrm{~nm}$, we used a sequence of 40 co-temporal and co-spatial IMaX (level-2) data (Figure 2A) to recognize the granules.

For our data cubes (SuFI and IMaX), a subsonic filter was used to suppress the global solar oscillations (e.g. five-minute p-modes) by attenuating modulations with horizontal speeds above $5 \mathrm{~km} \mathrm{~s}^{-1}$.

\section{Identification of Photospheric Features}

\subsection{Bright Points}

It has been found that the photospheric BPs have the highest brightness contrast in $214 \mathrm{~nm}$ images (Riethmüller et al., 2010). Riethmüller et al. (2010) have shown that sometimes the BP intensity reaches up to five times of the quiet-Sun mean intensity at this wavelength. Here, we present an automatic method for identifying BPs in images using Zernike moments and an SVM classifier. The following steps are employed:

Pixels with an intensity slightly lower than twice the mean intensity of the images are marked. These marked pixels are candidates for a final seed point. The function of region growing is exerted on the seed points. Region growing is an approach that on the basis of the brightness-threshold criterion makes it possible to group and segment the image pixels in large regions. Adjacent pixels of each seed that share certain characteristics with the seed join the initial seed points, and subsequently, the region grows $\left(R_{i}\right)$. The growth process terminates when all of the marked pixels from the previous step survive. In the final segmented images, the pixels of each region are separated (i.e., $R_{i} \cap R_{j}=\emptyset, i \neq j$ ) (Gonzalez 
and Woods, 2002). The intensity-weighted centroid, $\mathbf{r}_{C I}=\sum \mathbf{r}_{i} I\left(\mathbf{r}_{i}\right) / \sum I\left(\mathbf{r}_{i}\right)$, or the region's pixel nearest to the intensity-weighted centroid is selected as the final seed point. In the output of region growing, each region is labeled by a different integer number based on the Bwlabel function (Haralick and Linda, 1992). The output of the region-growing function on $214 \mathrm{~nm}$ image is shown in Figure 1B. These identified regions are grouped into the three main classes (individual BP, network BP, and non-BP).

In each training step, we probed (investigated) the data to select individual and network BPs (manually). The criteria for selecting the individual BPs (class 1) are their shapes, which would not be elongated and crescent-like structures located in the intergranular lanes compared with co-spatial and co-temporal $525 \mathrm{~nm}$ images. The size of these selected BPs often range from $8 \times 8$ pixels to $20 \times 20$ pixels. The mean value of their equivalent diameter is $\approx 0.3^{\prime \prime}$. The regions with the smaller or larger size are classified into two different classes: network BPs elongated in intergranular lanes, class 2 (Figure 1B, red rectangular boxes), and non-BPs, class 3 (Figure 1B, yellow circles). The Zernike moments of these three classes of image tiles are calculated, see Section 3.3. The normalized magnitudes of moments are given to the SVM classifier. This classifier, the robust supervised machine-learning algorithm, was designed based on the structuralrisk minimization (Vapnik, 1995). The purpose of this basically binary classifier is to find a decision boundary with a margin as large as feasible to reduce the classification error (Qu et al., 2003; Theodoridis and Koutroumbas, 2009).

Then, the classifier was applied to all labeled regions in all images. The code picks up a label 1 for an individual BP class, 2 for a network BP class, and 3 for a non-BP. When the individual BP or the network BP are classified, the size (number of pixels) and locations of the pixels are saved. Figure 1C shows the individual BPs and network BPs.

\subsection{Granules}

To detect granules, we used the $525.02 \mathrm{~nm}$ images. These images are segmented based on the mean shift procedure. This procedure is an old pattern-recognition approach in non-parametric feature-space analysis technique (Duda and Hart, 1973; Fukunaga and Hostetler, 1975). This feature-space analysis is the most useful and applicable tool for discontinuity-preserving smoothing, image filtering, and image segmentation. Discontinuity-preserving smoothing techniques were adapted to reduce both the smoothing amount near the abrupt changes at the edges and to remove the noise after a sufficiently many iterations. The mean shift image segmentation is a straightforward extension of the discontinuity-preserving smoothing algorithm (Comaniciu and Meer, 2002).

In Figure 2B, the segmented image is delineated. Similar to the BP training step (Section 3.1), the segmented regions are divided into two classes (granules and non-granular regions). The Zernike moments of these two classes (class 1, 2 ) of the image are computed and fed to the SVM classifier. The code is run for all images and picks up a label 1 for a granular class and 2 for a non-granular class. If it is a granule, the size and locations of pixels are saved. In Figure 2C, the identified regions are presented. 


\subsection{Zernike moments}

During the past decades, various moment functions (Hu, Legendre, Zernike, etc) have been proposed to describe images according to their ability to represent the image features based on orthogonal polynomials (Hu, 1962; Teague, 1980). The Zernike polynomials form a complete set of orthogonal polynomials over the interior of the unit circle, $x^{2}+y^{2} \leq 1$. The Zernike polynomial is given by

$$
\begin{aligned}
& V_{n}^{m}=R_{n}^{m}(r) \exp (i m \theta) \\
& R_{n}^{m}(r)=\sum_{s=0}^{(n-m) / 2}(-1)^{s} \frac{(n-s) !}{s !\left(\frac{n+|m|}{2}-s\right) !\left(\frac{n-|m|}{2}-s\right) !} r^{n-2 s},
\end{aligned}
$$

where the positive integer $n$ denotes the order number, and the repetition integer number $m$ that satisfies the constraint $n-|m|$ is even, and $|m| \leqslant n$ (Abandah and Anssari, 2009; Hosney, 2010).

Using the Zernike polynomials, the image intensity function $I(x, y)$ is expanded as

$$
I(x, y)=\sum_{n=0}^{n_{u p}} \sum_{m=0}^{n} Z_{n m} V_{n}^{m}(x, y) .
$$

The Zernike moments $Z_{n m}$ are obtained as

$$
Z_{n m}=\frac{n+1}{2} \iint_{\text {unit circle }} V_{n}^{m *}(x, y) I(x, y) d x d y .
$$

The orthogonal properties of Zernike polynomials enable the contribution of each Zernike moment $Z_{n m}$ to be unique and independent of the information of an image. Thus, the Zernike moments for each feature are unique (Hosney, 2010).

We calculated the Zernike moments of a maximum certain order up to $n_{u p}=$ 31. This gives a $(n, m)$ set, which is labeled from 1 to 528 . By testing different orders of Zernike moments, it was empirically realized that $n_{u p}=31$ maintained the quality of the reconstructed image (BP, granule, etc) with both polygonal and simple shapes when compared with the original one based on the normalized reconstruction error (Teh and Chin, 1988). Figure 3 displays one BP (upper panel) and one granule (lower panel) with their reconstructed images and plots of the normalized reconstruction errors as a function of order number. For both the reconstructed BP and granule, the normalized reconstruction error decreases with increasing order number and approaches 0.2 after $n \approx 9$. Empirically, the order number $n=31$ is chosen for the fast computations of the Zernike moments to support the convergence and robustness of the SVM classifier.

The main question is how Zernike moments extract the information of the features with various $n$ and $m$ numbers. For a unit circle $\left(x^{2}+y^{2} \leq 1\right)$ and $R_{n m}$ (Equation (1)), the weighted function becomes $r\left|R_{n m}(r)\right| \leq r$. The square of the image pixels that were mapped into the unit circle are closer to the circle perimeter and have more weight than the pixels located near the origin. The 
higher the order and repetition numbers, the more the Zernike functions oscillate. This gives us much confidence to describe the image based on corresponding moments.

To obtain scale and translation invariance, two methods are proposed: normalizing the image with respect to the regular geometrical moments before running the Zernike program (Khotanzad and Hong, 1990), and making radial Zernike moments invariant to scale and translation without the need to use the regular moments (Belkasim, Hassan, and Obeidi, 2004). We adopted the second method. The magnitude of the Zernike moments is invariant under rotation by nature. The pixels of image tiles are mapped into the unit circle. The Zernike moments of two individual BPs, two network BPs, and two non-BPs are shown in Figure 4. The Zernike moments of two granules and two non-granular regions are presented in Figure 5.

As shown in these figures, the magnitude values of the Zernike moments are clearly different for BPs and non-BPs. The BPs have a well-defined block structure. For non-BP features, we see some block structures, but they are different from those of the BPs. These differences give us much confidence in applying the SVM classifier to identify the photospheric features from in the $214 \mathrm{~nm}$ images.

We created some artificial data (mimicking BPs) to describe the behavior of the Zernike moments. Artificial BPs are generated by using a two-dimensional Gaussian function,

$$
\begin{aligned}
B(x, y)=A \exp ( & \left.-\left(a\left(x-x_{0}\right)^{2}+2 b\left(x-x_{0}\right)\left(y-y_{0}\right)+c\left(y-y_{0}\right)^{2}\right)\right), \\
a & =\cos ^{2} \theta / 2 \sigma_{x}^{2}+\sin ^{2} \theta / 2 \sigma_{y}^{2}, \\
b & =-\sin 2 \theta / 4 \sigma_{x}^{2}+\sin 2 \theta / 4 \sigma_{y}^{2}, \\
c & =\sin ^{2} \theta / 2 \sigma_{x}^{2}+\cos ^{2} \theta / 2 \sigma_{y}^{2}
\end{aligned}
$$

where $A$ is the amplitude, $x_{0}, y_{0}$ are the positions of the center of the peak, $\sigma_{x}, \sigma_{y}$ are controlled width (FWHM) of the $\mathrm{BP}$, and $\theta$ is the angle measured clockwise.

In Figure 6, the Zernike moments of artificial BPs (for different amplitudes and widths) are computed. The structures of the Zernike moments for faint artificial BP $(A=10)$ and bright artificial BP $(A=100)$ are the same. The structures of moments with different widths $\left(\sigma_{x}=\sigma_{y}=2.5,3,3.5,4\right)$ do not change significantly. As expected, our calculations show that for $\sigma_{x} \neq \sigma_{y}$, with changing rotation angles, the structure of the moments are the same. Furthermore, the Zernike moments of the overlapping artificial BPs and mimicking network BPs with different arrangements appeared as similar block structures (Figure 7).

Additionally, the Zernike moments with higher order numbers are sensitive to noise (e.g., Teh and Chin, 1988). Both our training and test (for BPs and granules) sets that include noisy image tiles change the structure of the moments so slightly that SVM classifier is able to distinguish them. 


\section{Results}

By applying our automatic detection to SuFI images, about 1000 BPs were recognized. There are two groups of BPs that have been attained by our code: about $75 \%$ are individual BPs, most of which can be mapped on a square with an equivalent diameter of $\approx 0.3^{\prime \prime}$; the remaining $25 \%$ are network BPs.

The size distribution of BPs and their filling factor (coverage area in each image) are shown in Figure 8. The power-law distribution $\left(N \propto A^{-\alpha}\right)$ is fitted with a power exponent $\alpha \approx 1.5$ (Figure 8 , upper panel). The filling factor of BPs per image with mean values of 0.01 is presented (Figure 8 , lower panel).

The brightness fluctuations and the scatter plot of BPs are shown in Figure 9. The brightness fluctuations relative to the intensity of the photosphere are given by

$$
I_{\text {fluc }}=\sqrt{\frac{\sum(I-\bar{I})^{2}}{N \bar{I}^{2}}},
$$

where $\bar{I}$ is the mean intensity of the photosphere and $N$ is the number of pixels (Roudier and Muller, 1987; Yu et al., 2011). As we see, the brightness fluctuations of BPs (Figure 9, left panel) are larger than 1.1. As expected, the BP regions are brighter than their surrounding areas. The average of the brightness fluctuations in SuFI images is about 0.1. Increasing the size of BPs will subsequently increase the scatter in their average brightness (Figure 9, right panel). For network BPs $\left(\geq 0.2 \operatorname{arcsec}^{2}\right)$, the brightness is more widely scattered. The latter could be related to the magnetic pressure inside flux tubes, combined with the lateral extension, inclination angle, and opacity.

An individual $\mathrm{BP}$ and an individual $\mathrm{BP}$ within the network were tracked in seven time steps (each 234 second long). They were included in the image tiles and the function of region growing was exerted on them. We used the function align_cube_correl.pro, which is available in the SSW/IDL package, to coalign the images in the sequence. The intensity-weighted centroid was then computed for a sequence of the segmented image tiles (Figure 10). The horizontal velocity of the centroid, $v_{C I}=\Delta \mathbf{r}_{C I} / \Delta t$, for both consecutive frames (with $\Delta t \simeq 39$ seconds) was calculated and the mean value of velocity was derived (see, e.g., Jafarzadeh et al., 2013). The mean horizontal velocities of the individual BP and the individual one integrated within the network $\mathrm{BP}$ are $v_{\mathrm{IBP}}=1.6 \pm 0.3 \mathrm{~km}$ $\mathrm{s}^{-1}$ and $v_{\mathrm{IBPN}}=0.9 \pm 0.5 \mathrm{~km} \mathrm{~s}^{-1}$, respectively. These results are consistent with previous studies $\left(1-2 \mathrm{~km} \mathrm{~s}^{-1}\right)$ obtained by Möstl et el. (2006) and Jafarzadeh et al. (2013).

The excitation of the kink waves in the flux tubes of BPs might be related to horizontal motions (Choudhuri, Auffret, and Priest, 1993; Muller et al., 1994; Wellstein, Kneer, and von Uexküll, 1998; Jafarzadeh et al., 2013). The energy flux, carried by the excitation of the kink waves, is proportional to the square of the horizontal velocity, lifetime, filling factor, scale height and density of BPs. The proportion of the energy flux of the individual BP $\left(E_{\mathrm{IBP}}\right)$ to the network $\mathrm{BP}\left(E_{\mathrm{IBPN}}\right)$ with almost the same filling factors and lifetimes is given by

$$
\frac{E_{\mathrm{IBP}}}{E_{\mathrm{IBPN}}}=\frac{v_{\mathrm{IBP}}^{2}}{v_{\mathrm{IBPN}}^{2}}=3.16 \pm 2.32 .
$$


It shows that the contribution of an individual BP to the transfer of the energy to the upper layers is more than that of an individual BP into the network.

Figure 11 illustrates the size distribution of the granules and their filling factor (coverage area in each image). The peak value of the size distribution is held on $0.5 \operatorname{arcsec}^{2}$ with an equivalent diameter of $0.8 \operatorname{arcsec}$ (Figure 11, left panel). The two-parameter lognormal distribution with $\sigma=0.7$ and $\mu=5.9$ is fitted. The equivalent diameter is often concentrated on an interval of $0.6-2.5^{\prime \prime}$. The mean value of the filling factors of the granules equals 0.51 (Figure 11, right panel). The difference between the filling factor in image number 21 and the total average was found to be $7 \%$. Our computations show that the average brightness of these two images is about $8 \%$ lower than the overall average (average brightness of 40 images).

The brightness fluctuations and the scatter plot of granules are shown in Figure 12. The mean value of the brightness fluctuations of the granules (Figure 12 , left panel) is about 0.22 . The granules with areas $\leq 2.5 \operatorname{arcsec}^{2}$ with an equivalent diameter shorter than 1.7 arcsec have excessive scatter in brightness (with large brightness fluctuations) and this scatter in brightness decreases with increasing granule size (Figure 12, right panel). For large granules, the brightness approaches unity.

To estimate the reliability of the algorithm, we visually inspected 200 of the detected BPs. We found that 18 of them were not clear BPs. Using a constant threshold for all of the images seemed to be the cause of erroneous detections. In fact, background brightness, noises, etc, are different from image to image. The invariant Zernike moments can detect BPs even if they are small or have variable brightness (Alipour, Safari, and Innes, 2012). Because of the above-mentioned reasons, false-positive detections appeared when BPs are recognized.

As we know, BPs are ubiquitously observed in intergranular lanes and their locations are correctly identified. We compared recognized BPs in SuFI images with co-temporal and co-spatial IMaX magnetograms (Figure 13). As shown in Figure 13B, the identified BPs show positive and/or negative polarities in the magnetogram (Figure 13A, red box).

The recognition of the granules has an efficiency slightly better than $85 \%$, but regarding the intergranular regions, we obtain almost $80 \%$ of the answers correctly. The error in the latter result arises because of the heterogeneous shapes of the intergranular lanes; consequently, as a result of an oversegmentation in these areas, distinguishing between the regions would be somewhat difficult for the classifier.

\section{Conclusions}

We presented an automated detection and characterization of photospheric BPs and granules. Image-processing methods and a machine-learning algorithm were applied to recognize and determine the physical properties of these features. The processes of two kinds of image segmentation and the invariant Zernike moments were employed. The structure of the Zernike moments for both faint and bright 
features with different sizes and various orientations are distinctive enough (Figures 6 and 7) to be identified using the SVM. The entire computing time has taken less than one hour for each image. BPs identified by our algorithm were located in the intergranular regions, as a comparison with IMaX data revealed. If the emergence and existence of BPs is taken as a criterion for the level of magnetic activity, it can be suggested that these images represented the quietSun because the filling factor of BPs in such data is much lower than $5 \%$ (Andic et al., 2011). With respect to the set of horizontal velocities of the photospheric $\mathrm{BPs}$, it seems that energy transfer to the upper layers frequently returns to the individual BPs rather than the individual BPs within the network.

Apparently, two regimes govern the relationship between the granules brightness and their sizes. Granules with sizes smaller than $2.5 \operatorname{arcsec}^{2}$, the critical scale, are more scattered in brightness, and for larger granules, the brightness approaches unity. This behavior of small granules can be attributed to the nature of their turbulent eddies, while large granules are considered as convective elements (Roudier and Muller, 1987; Yu et al., 2011).

Our code is extendable for the detection of both surface and atmospheric solar features (e.g., sunspots, flares, and CMEs) observed by the ground-based telescopes (e.g., SST, NST, GREGOR; and with future instruments ATST, EST, Indian NLST). Finally, the algorithm will be improved to track BPs and the granules in a sequence of images.

Acknowledgements The German contribution to Sunrise is funded by the Bundesministerium für Wirtschaft und Technologie through the Deutsches Zentrum für Luft- und Raumfahrt e.V. (DLR), Grant No.50 OU 0401, and by the Innovationsfonds of the President of the Max Planck Society (MPG). The Spanish contribution has been funded by the Spanish MICINN under projects ESP2006-13030-C06 and AYA2009-14105-C06 (including European FEDER funds). HAO/NCAR is sponsored by the National Science Foundation, and the HAO Contribution to Sunrise was partly funded through NASA grant number NNX08AH38G. The authors thank Julian Blanco and Valentin Martínez Pillet for providing access to IMaX data and Sami K. Solanki for helpful comments and discussions. The authors thank the anonymous referee for meticulous comments and suggestions.

\section{References}

Abandah, G., Anssari, N.: 2009, Journal of Computrer Science 5, 3, 226.

Aipour, N., Safari, H., Innes, D. E.: 2012, Astrophys. J. 746, 12.

Andic, A., Chae, J., Goode, P. R., Cao, W., Ahn, K., Yurchyshyn, V., Abramenko, V.: 2011, Astrophys. J. 731, 29.

Aschwanden M. J.: 2010, Solar Phys. 262, 235.

Barthol, P., Gandorfer, A., Solanki, S. K., Schüssler, M., Chares, B., Curdt, W., et al.: 2011, Solar Phys. 268, 1.

Belkasim, S., Hassan, E., Obeidi, T.: 2004, Computer and Information Technology, 790, 4.

Berger, T. E., Scherijver, C. J., Shine, R. A., Tarbell, T. D., Title, A. M., Scharmer, G.: 1995, Astrophys. J. 454, 531 .

Berkefeld, T., Schmidt, W., Soltau, D., Bell, A., Doerr, H. P., Feger, B., et al.: 2011, Solar Phys. 268, 1.

Bovelet, B., Wiehr, E.: 2007, Solar Phys. 243, 121.

Choudhuri, A. R., Auffret, H., Priest, E. R.: 1993, Solar Phys. 143, 49.

Cloutman, L. D.: 1979, Astrophys. J. 227, 614.

Comaniciu, D., Meer, P.: 2002, IEEE Trans. Pattern Anal. Machine Intell. 24, 603.

de Wijn, A. G., Stenflo J. O., Solanki, S. K., Tsuneta, S.: 2008, Space Sci. Rev. 144, 275. 
Duda, R. O., Hart, P. E.: 1973, Pattern Classification and Scene Analysis., Wiley.

Dunn, R. B., Zirker, J. B.:1973, Solar Phys. 33, 281.

Frazier, E. N., Stenflo, J.O.: 1978, Astron. Astrophys. 70, 789.

Fukunaga, K., Hostetler, L. D.: 1975, IEEE Trans. Information Theory. 21, 32.

Gandorfer, A., Grauf, B., Barthol, P., Riethmüller, T. L., Solanki, S. K., Chares, B., et al.: 2011, Solar Phys. 268, 1.

Gonzalez, R. C., Woods, R. E.: 2002, Digital Image Processing, 2nd edn., Pearson Prentice Hall, Upper Saddle River.

Haralick, R. M., Linda, G. S.: 1992, Computer and Robot Vision, Volume I, Addison-Wesley, pp. $28-48$.

Hosney, K. M.: 2010, Information Sciences, 180, 2299.

Hu, M. K.: 1962, IRE Trans. Inf. Theory, IT-8, 179.

Jafarzadeh, S., Solanki, S. K., Feller, A., Lagg, A., Pietarila, A., Danilovic, S., et al.: 2013, Astron. Astrophys. 549, 116.

Khotanzad, A., Hong, Y. H.: 1990, IEEE Trans. Pattern Anal. Machine Intell. 12, 5.

Kobel, P., Hirzberger, J., Solanki, S. K., Gandorfer, A., Zakharov, V.: 2010, Astron. Astrophys. 502, 303.

Martínez Pillet, V., del Toro Iniesta, J. C., Álvarez-Herrero, A., Domingo, V., Bonet, J. A., González Fernández, L., et al.: 2011, Solar Phys. 268, 1.

Möstl, C., Hanslmeier, A., Sobotka, M., Puschmann, K., Muthsam, H. J.: 2006, Solar Phys. 237, 13.

Muller, R., Roudier, Th., Vigneau, J., Auffret, H.: 1994, Astron. Astrophys. 283, 232.

Nordlund, A., Stein, R. F., Asplund, M.: 2009, Living Rev. Solar Phys. 6, 2.

Qu, M., Shih, F. Y., Jing, J., Wang, H.: 2003, Solar Phys. 217, 157.

Riethmüller, T. L., Solanki, S. K., Martínez Pillet, V., Hirzberger, J., Feller, A., Bonet, J. A., et al.: 2010, Astrophys. J. Lett. 723, 169.

Robitaille, P. M.: 2011, Progress in Physics, 3, 79

Roudier, T., Muller, R.: 1987, Solar Phys. 107, 11.

Rusu, M.: 2005, PADEU, 15, 59.

Sánchez Almeida, J., Márquez, I., Bonet, J. A., Domínguez Cerdeña, I., Muller, R.: 2004, Astrophys. J. 609, 91.

Solanki, S. K., Barthol, P., Danilovic, S., Feller, A., Gandorfer, A., Hirzberger, J., et al.: 2010, Astrophys. J. 723, 127 .

Stein, R. F.: 2012, Living Rev. Solar Phys. 9, 4

Teague, M. R.: 1980, J. Opt. Soc. Am., 70, 920

Teh, C., Chin, R. T.: 1988, IEEE Trans. Pattern Anal. Machine Intell. 10, 4.

Theodoridis, S., Koutroumbas, K. : 2009, Pattern Recognition, 4th edn., Academic Press, Elsevier Inc.

Utz, D., Hanslmeier, A., Möstl, C., Muller, R., Veronig, A., Muthsam, H.: 2009, Astron. Astrophys. 498, 289.

Vapnik, V. N.: 1995, The nature of statistical learning theory. Berlin Heidelberg, New York: Springer-Verlag.

Wellstein, S., Kneer, F., von Uexküll, M.: 1998, Astron. Astrophys. 335, 323.

Yu, D., Xie, Z., Hu, Q., Yang, S., Zhang, J., Wang, J.: 2011, Astrophys. J. 743, 58.

Zhang, H., Scharmer, G., Lofdahl, M., Yi, Z.: 1998, Solar Phys. 183, 283. 


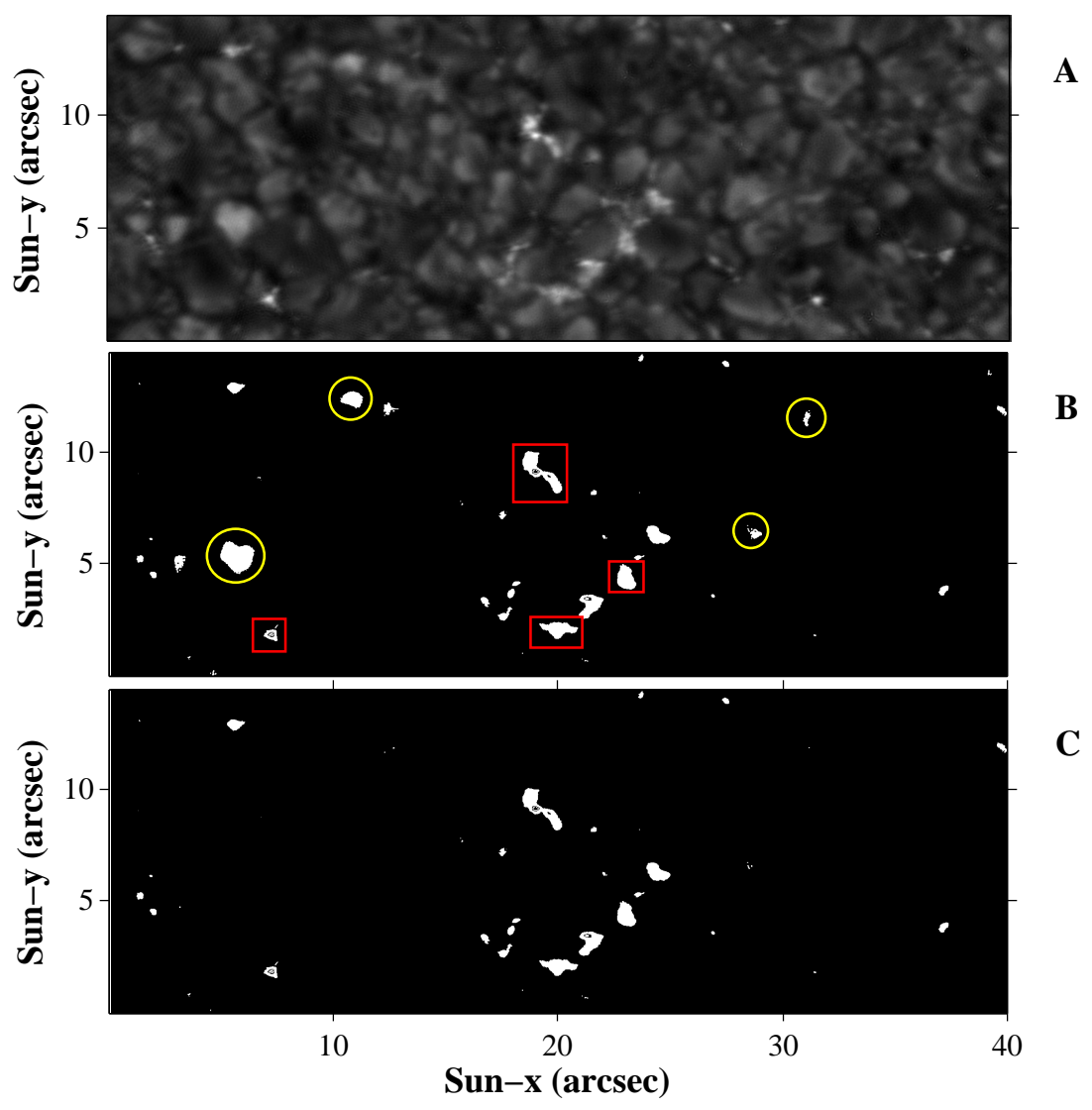

Figure 1. A Sunrise/SuFI $214 \mathrm{~nm}$ image recorded on 9 June 2009 (14:44:03 UT) (A), the output of the region growing function (B); samples of network BPs and non-BPs are indicated by red rectangular boxes and yellow circles, respectively. The output of the SVM classifier for identification of BPs $(\mathrm{C})$. 


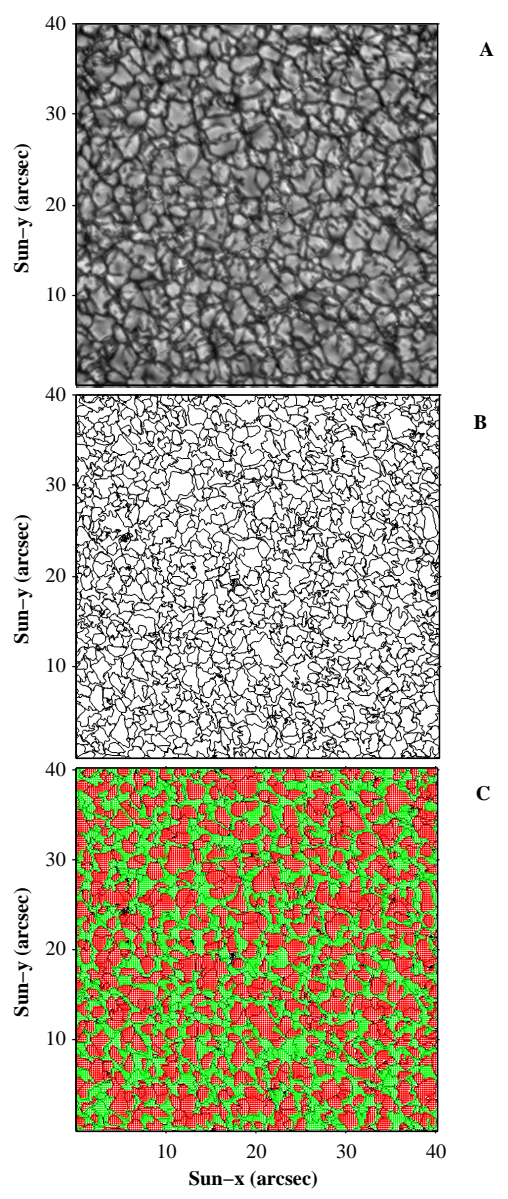

Figure 2. A Sunrise/IMaX $5250.02 \mathrm{~nm}$ image recorded on 9 June 2009 (14:27:14 UT) (A), the segmented image based on mean shift procedure (B), the output of the SVM classifier (C). Red and green regions are representative of granules and non-granular regions, respectively. 


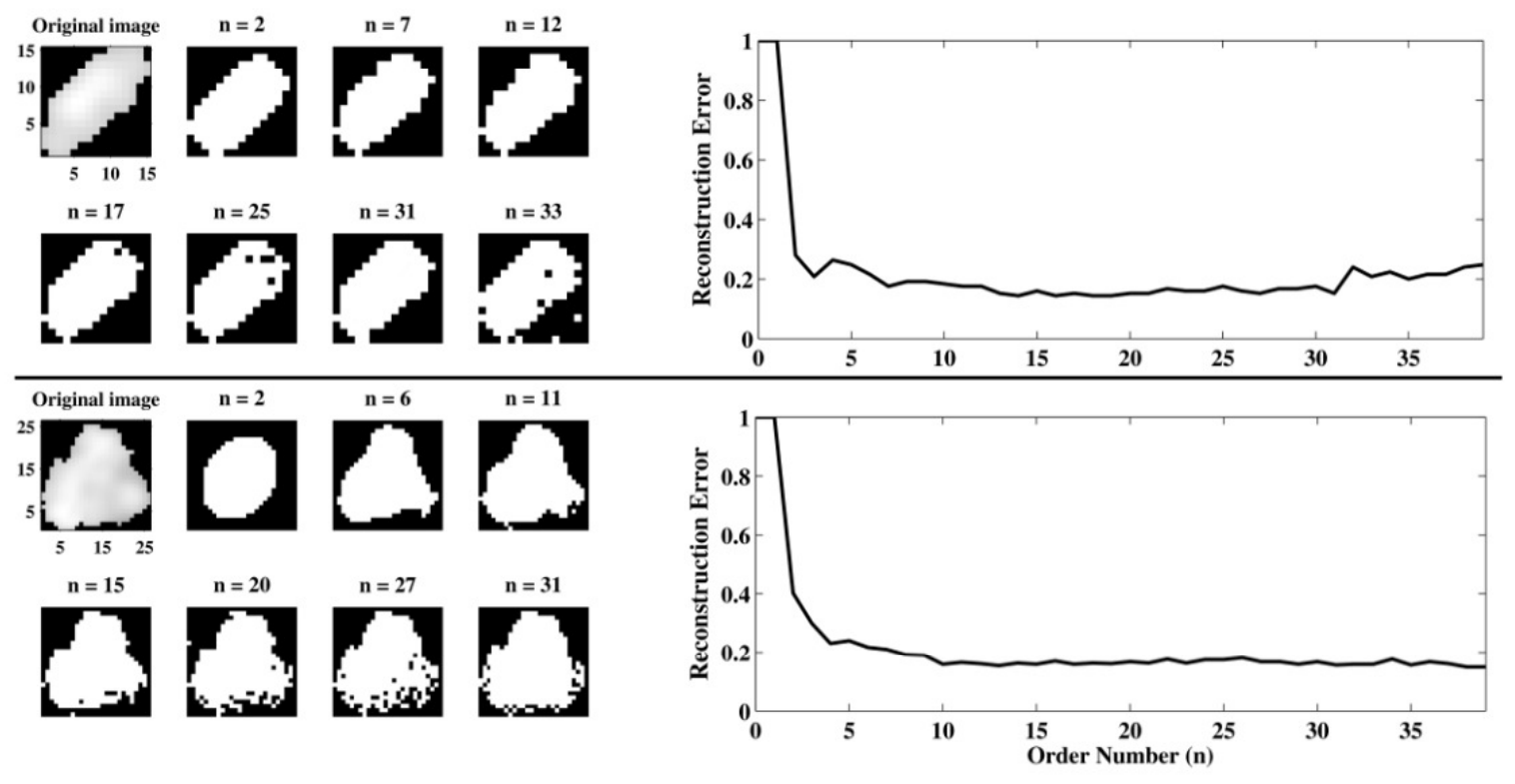

Figure 3. Reconstructed image of a BP (binary map) with different numbers of $n$ (left, top). The normalized reconstruction error, $e^{2}(n)=\frac{\sum_{i} \sum_{j}\left[f(i, j)-\hat{f}_{n}(i, j)\right]^{2}}{\sum_{i} \sum_{j}[f(i, j)]^{2}}$, in which $f(i, j)$ is the image function and $\hat{f}_{n}(i, j)$ is the reconstructed version, is plotted (right, top). The reconstructed image of a granule (binary map) with different numbers of $n$ is displayed (left, bottom). The normalized reconstruction error is plotted (right, bottom). The normalized reconstruction error decreases with increasing order number and approaches 0.2 after $n \approx 9$ (see text). 


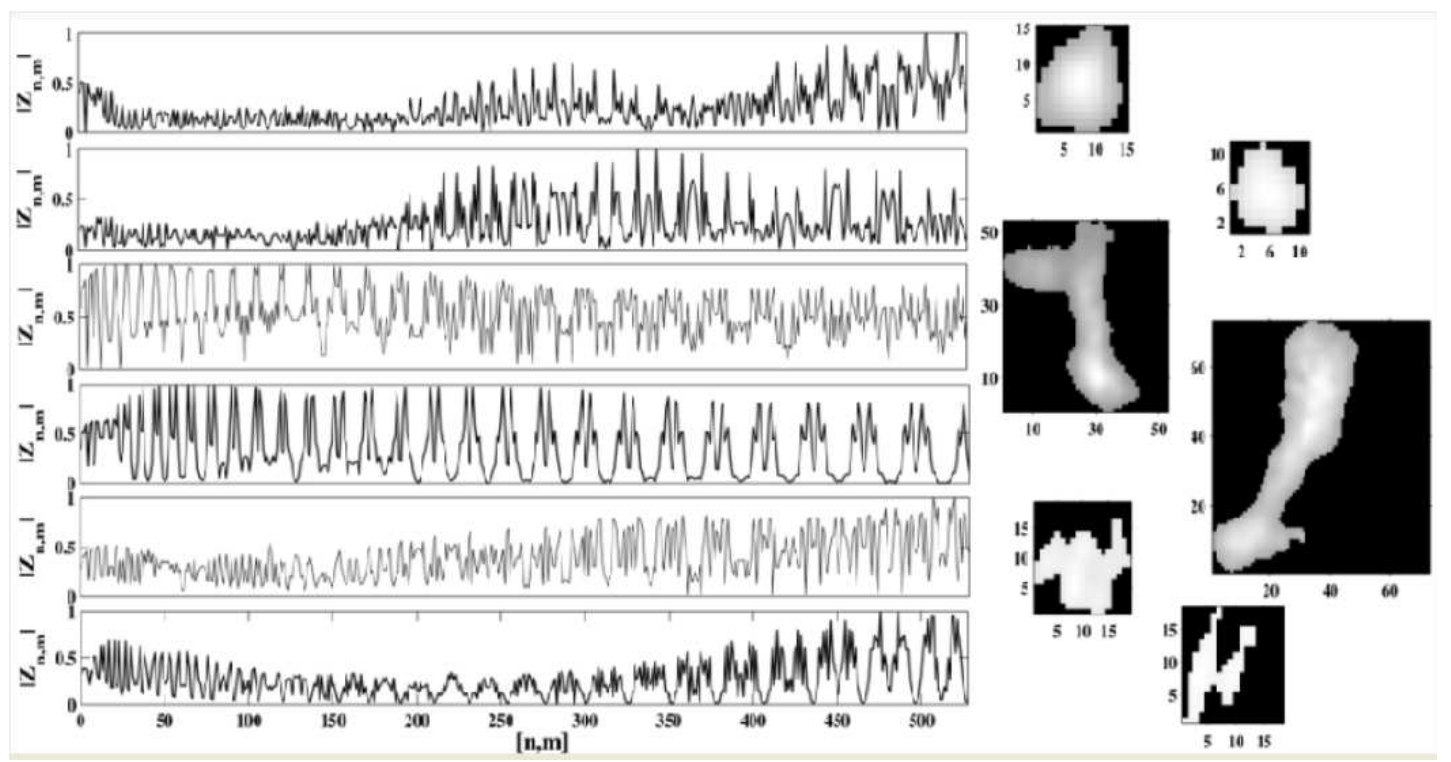

Figure 4. Magnitude values of Zernike moments, $\left|Z_{n m}\right|$, of two BPs (first and second rows), two network BPs (third and fourth rows), and two non-BPs (penultimate and last rows). The corresponding features are displayed on the right. 


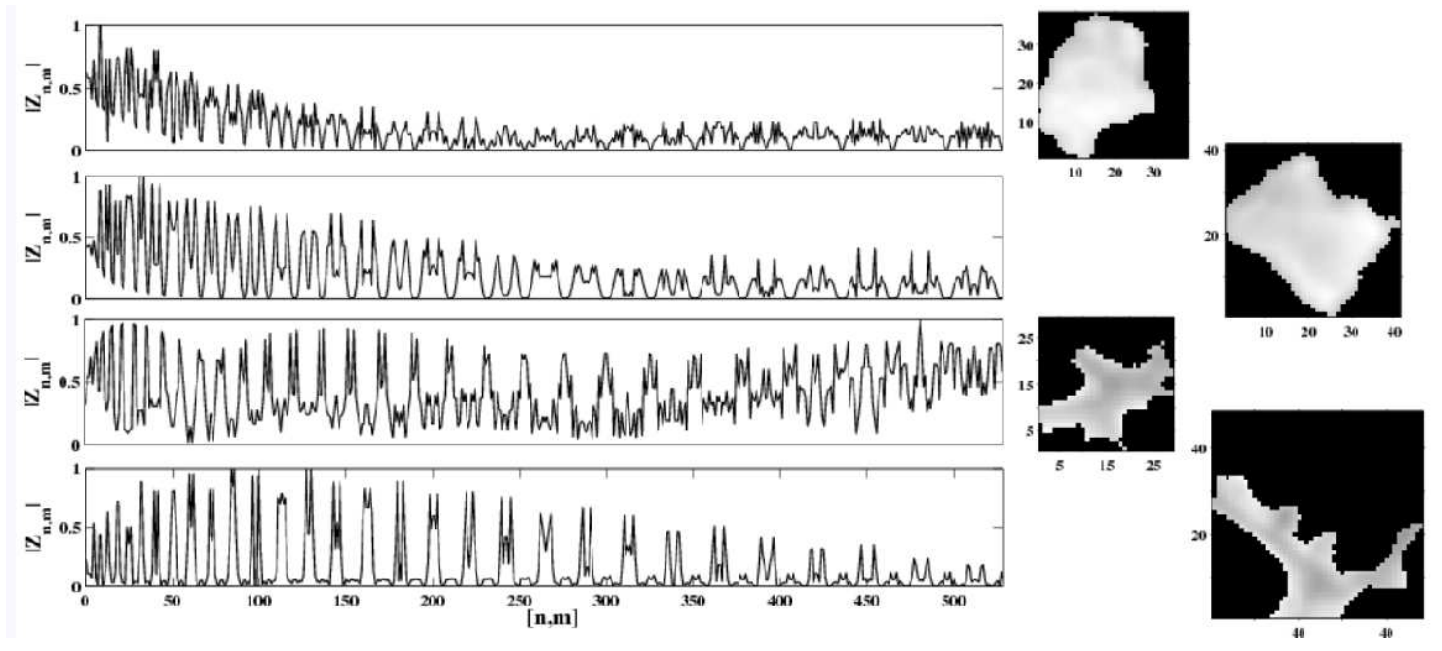

Figure 5. Magnitude values of Zernike moments, $\left|Z_{n m}\right|$, of two granules (first and second rows) and non-granular regions (third and last rows). The corresponding features are displayed on the right. 

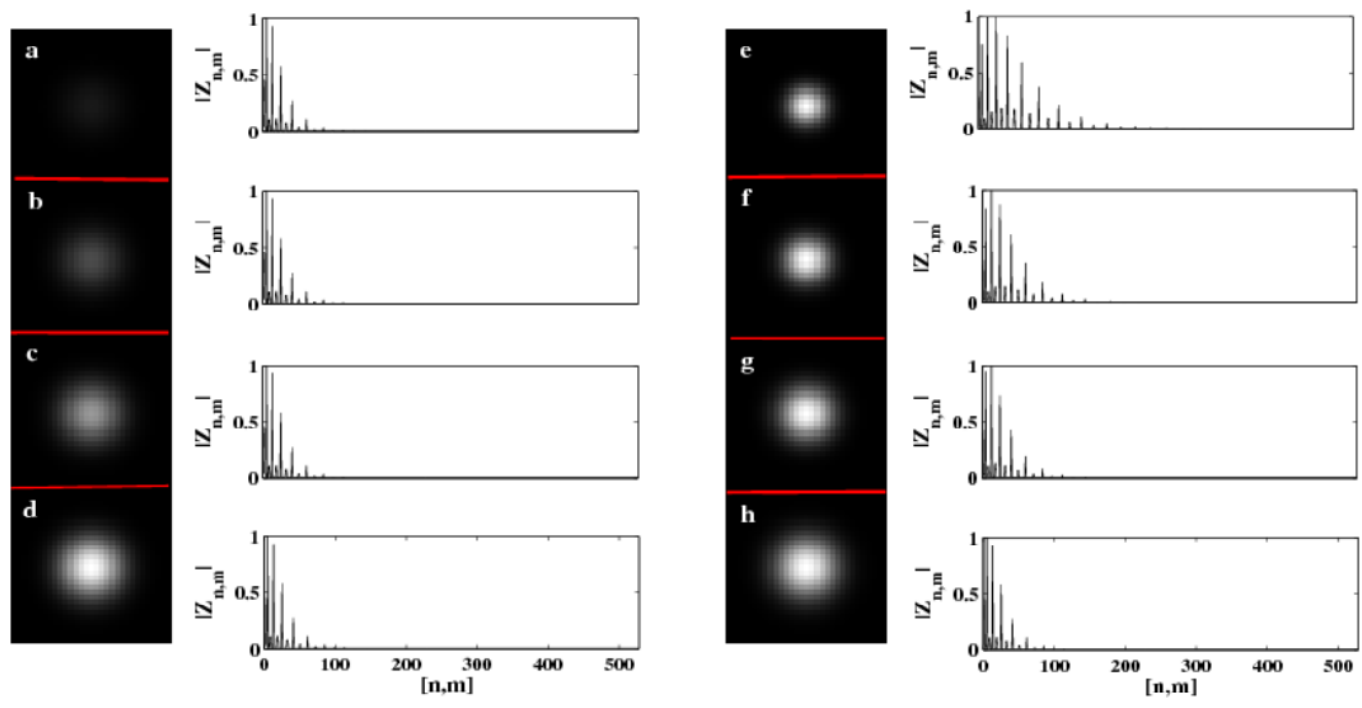

Figure 6. Artificial BPs with (a) $A=10$, (b) $A=30$, (c) $A=60$, (d) $A=100$ and the auxiliary parameters are $\sigma_{x}=\sigma_{y}=4, x_{0}=y_{0}=0, \theta=0$ (left); artificial BPs with (e) $\sigma_{x}=\sigma_{y}=2.5$, (f) $\sigma_{x}=\sigma_{y}=3$, (g) $\sigma_{x}=\sigma_{y}=3.5$, (h) $\sigma_{x}=\sigma_{y}=4$ and the auxiliary parameters are $A=50, x_{0}=y_{0}=0, \theta=0$ (right). 

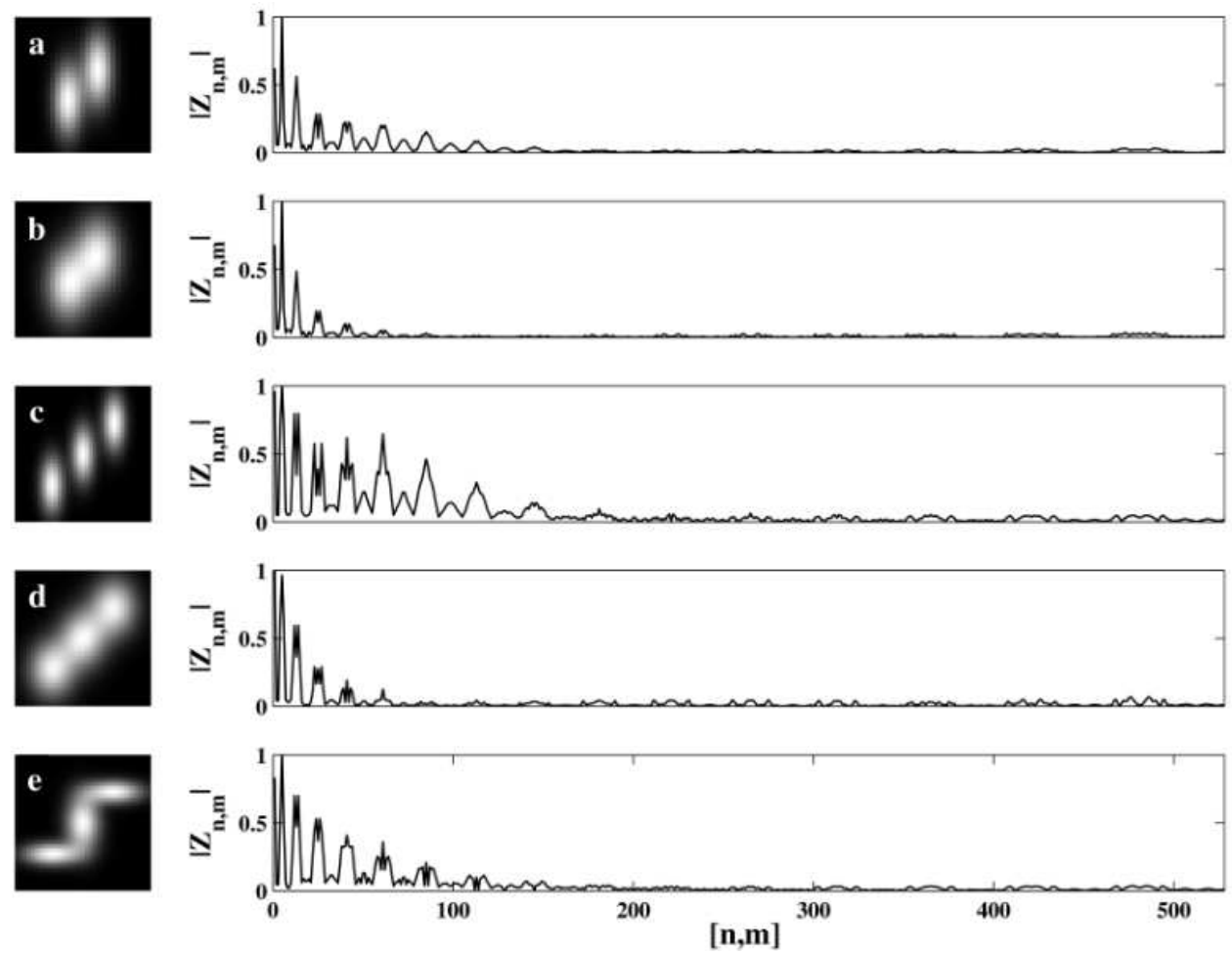

Figure 7. Artificial BPs with (a) $x_{0}=y_{0}=-3, x_{1}=y_{1}=3, \sigma_{x_{0}}=\sigma_{x_{1}}=2, \sigma_{y_{0}}=\sigma_{y_{1}}=5$; (b) $x_{0}=y_{0}=-3, x_{1}=y_{1}=3, \sigma_{x_{0}}=\sigma_{x_{1}}=3, \sigma_{y_{0}}=\sigma_{y_{1}}=5$; (c) $x_{0}=y_{0}=-8$, $x_{1}=y_{1}=0, x_{2}=y_{2}=8, \sigma_{x_{0}}=\sigma_{x_{1}}=\sigma_{x_{2}}=2, \sigma_{y_{0}}=\sigma_{y_{1}}=\sigma_{y_{2}}=5$; (d) $x_{0}=y_{0}=-8$, $x_{1}=y_{1}=0, x_{2}=y_{2}=8, \sigma_{x_{0}}=\sigma_{x_{1}}=\sigma_{x_{2}}=4, \sigma_{y_{0}}=\sigma_{y_{1}}=\sigma_{y_{2}}=5$; (e) $x_{0}=y_{0}=-8$, $x_{1}=y_{1}=0, x_{2}=y_{2}=8, \sigma_{x_{0}}=\sigma_{x_{2}}=\sigma_{y_{1}}=5, \sigma_{x_{1}}=3, \sigma_{y_{0}}=\sigma_{y_{2}}=2$ and the auxiliary parameters are $A=10, \theta=0$. 

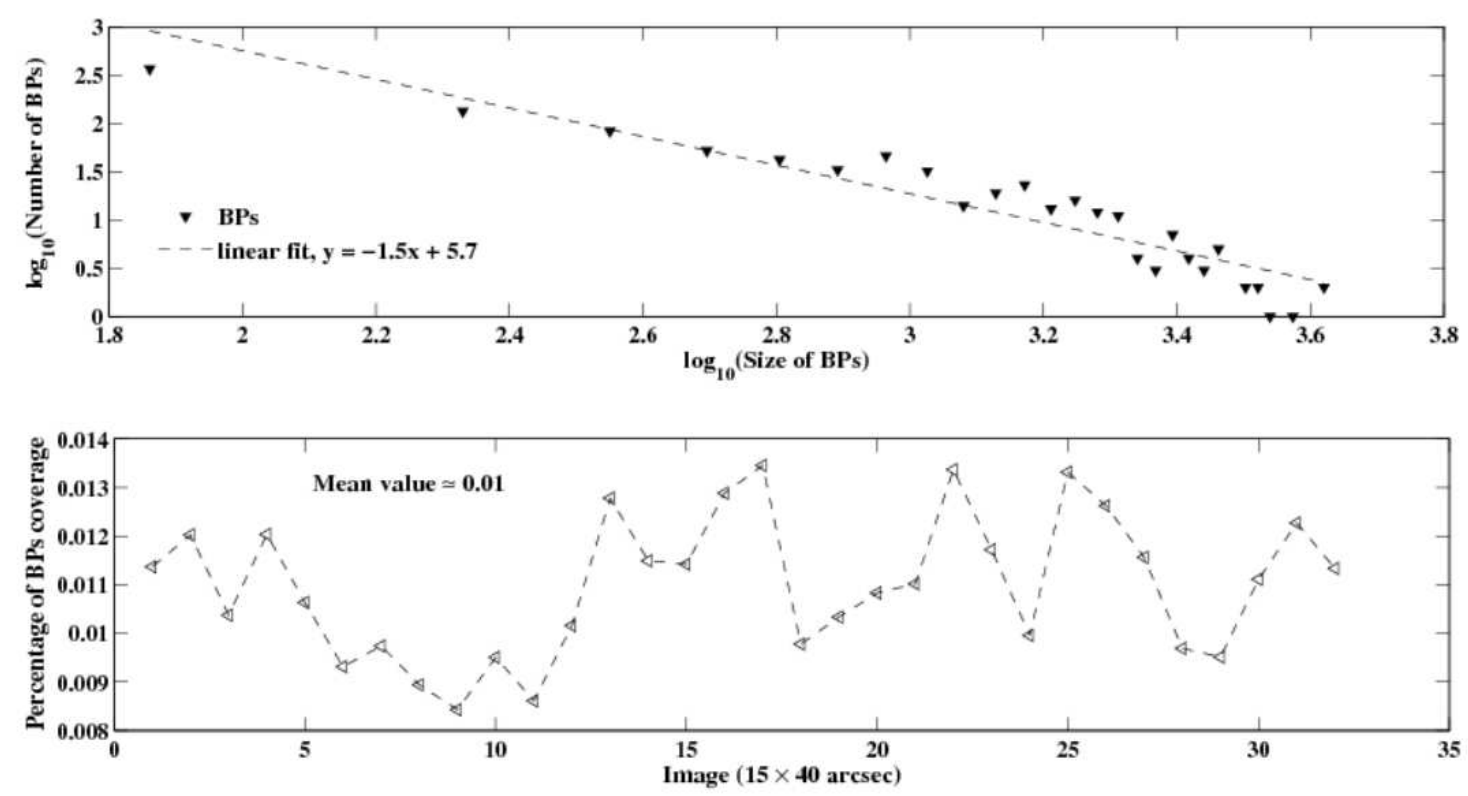

Figure 8. The size distribution of BPs detected in SuFI images. The power-law fit, $N \propto A^{-\alpha}$, is presented on a $\log$ scale with $\alpha \approx 1.5$ (upper panel). The filling factor of BPs per image with mean values of 0.01 is shown (lower panel). 

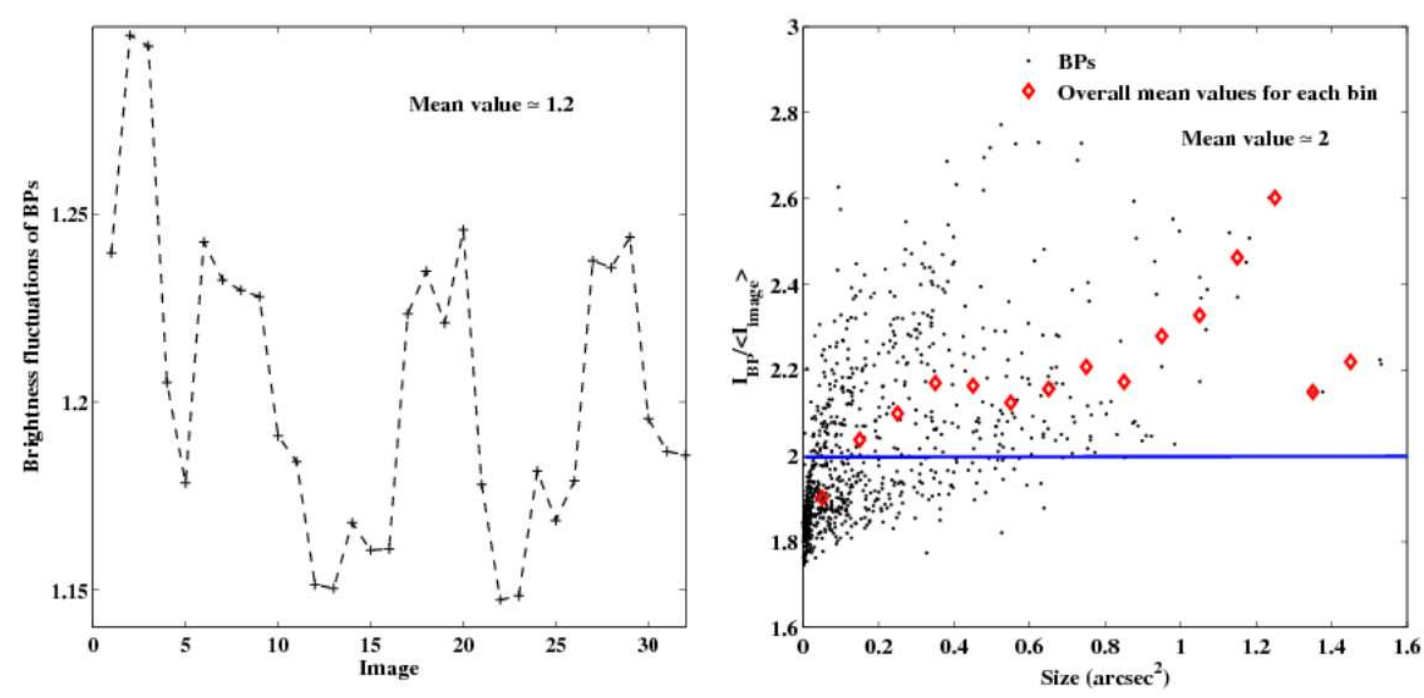

Figure 9. Brightness fluctuations of BPs (left panel) and scatter plots of BP brightness vs. size (right panel) are shown. The mean value of the scatter plots of the BP brightness is about 2. The overall mean values for each bin (0-0.1 arcsec, $0.1-0.2$ arcsec, $0.2-0.3$ arcsec, etc) are presented as red diamonds $(\diamond)$. 


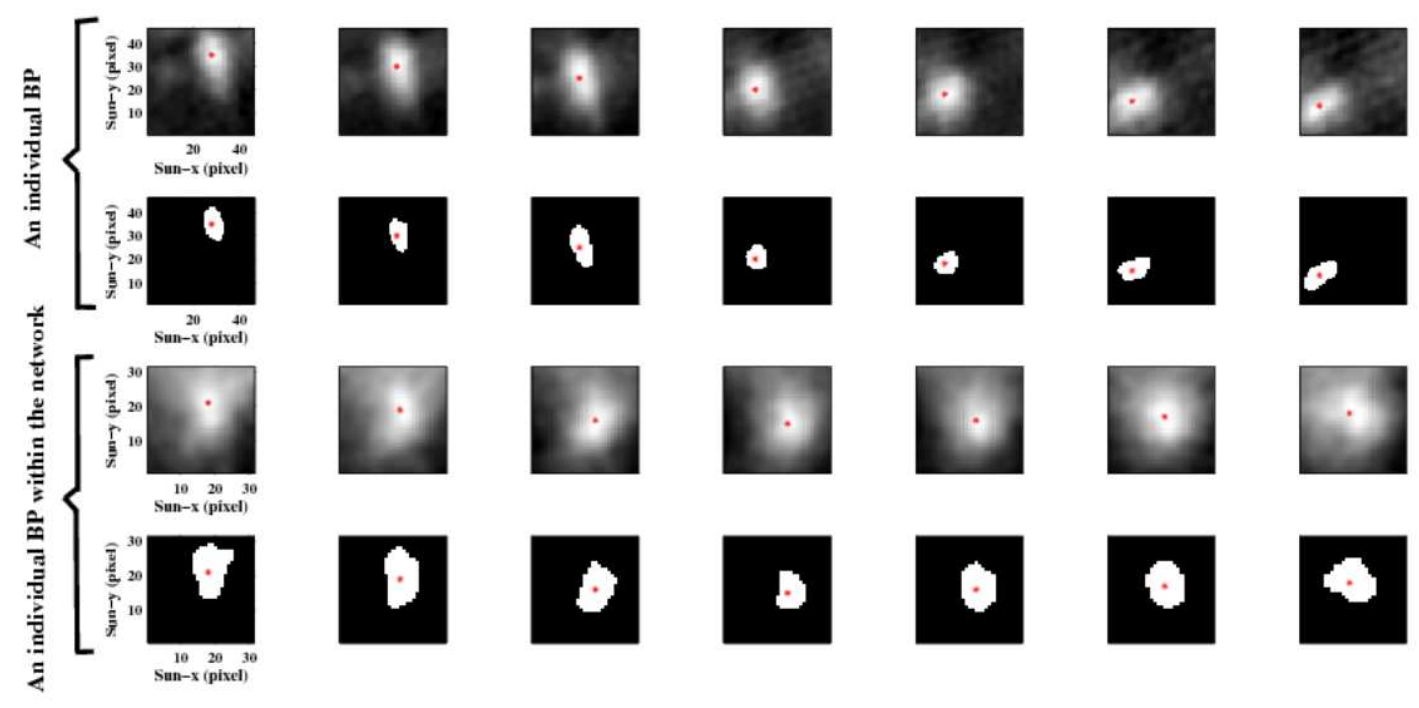

Figure 10. A consecutive list of image tiles including an individual BP, recorded from 14:55:45 to 14:59:39 UT on 9 June 2009, is shown in the first row. The result of applying the function of region growing on the sequence of the first-row images is represented as segmented images in the second row. In the third row, a network BP is displayed in a sequence of subframes recorded from 14:33:39 to 14:37:33 UT on 9 June 2009. In the last row, the segmented images are obtained by applying the function of region growing to a sequence of third-row images. The red point represents the intensity-weighted centroid of pixels in the original image obtained from segmentation. 

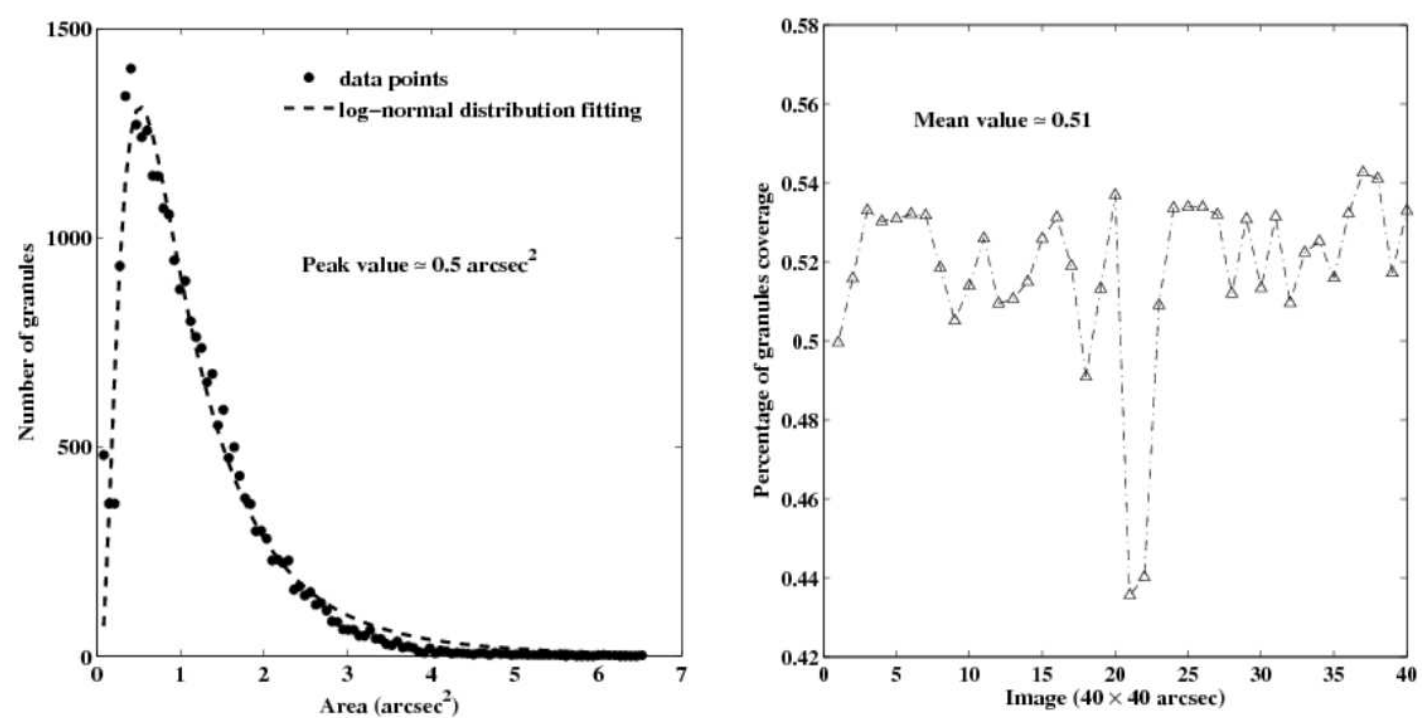

Figure 11. The size distribution of granules detected in IMaX images is shown (left panel). The peak value is about $0.5 \operatorname{arcsec}^{2}$. A two-parameter lognormal function, $\frac{1}{x \sigma \sqrt{2 \pi}} \exp \left(-\frac{(\ln x-\mu)^{2}}{2 \sigma^{2}}\right)$, with $\mu=5.908$ and $\sigma=0.7$ is fitted (dashed line). The filling factor of granules per image with mean values of 0.51 is shown (right panel). 

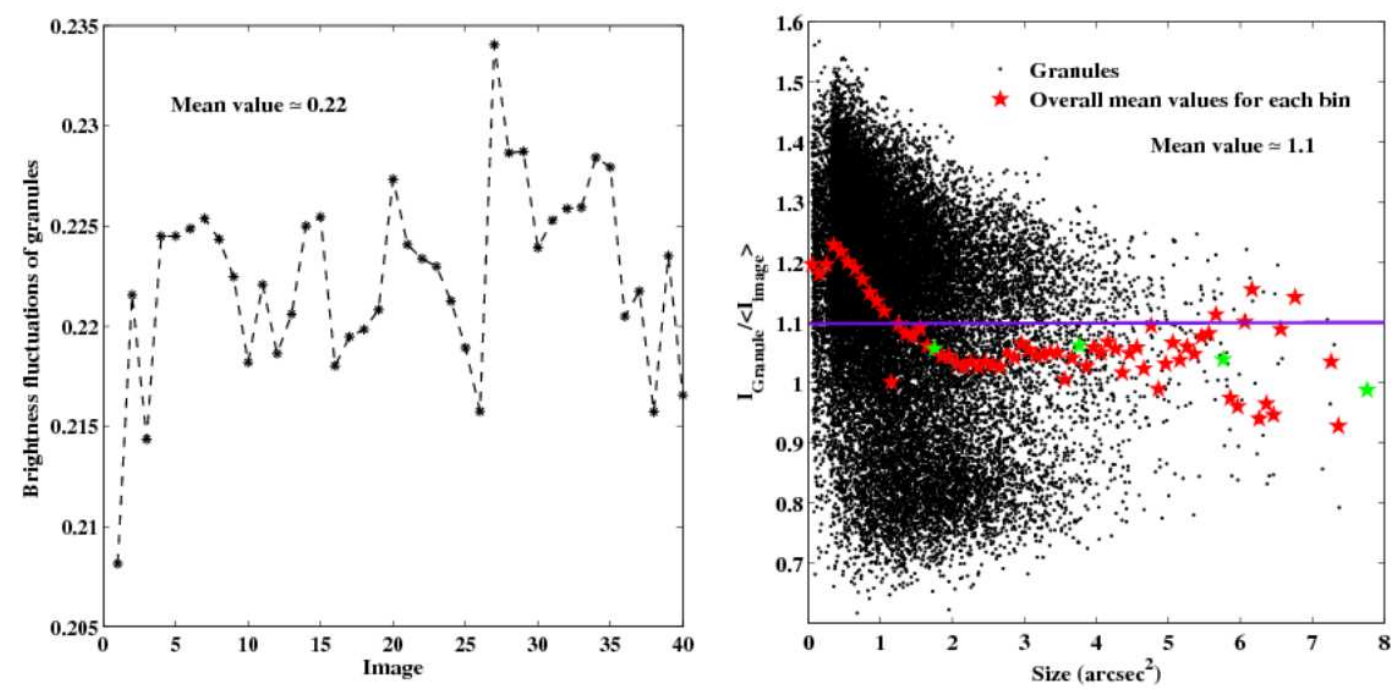

Figure 12. Brightness fluctuations of granules (left panel) and a scatter plot of granules brightness vs. size (right panel). The overall mean value of the scatter plot of the granule brightness is 1.1. For large granules, the brightness approaches unity. The mean values for each bin (0-0.1 arcsec, $0.1-0.2$ arcsec, $0.2-0.3$ arcsec, etc) are shown as red stars $(\star)$. 


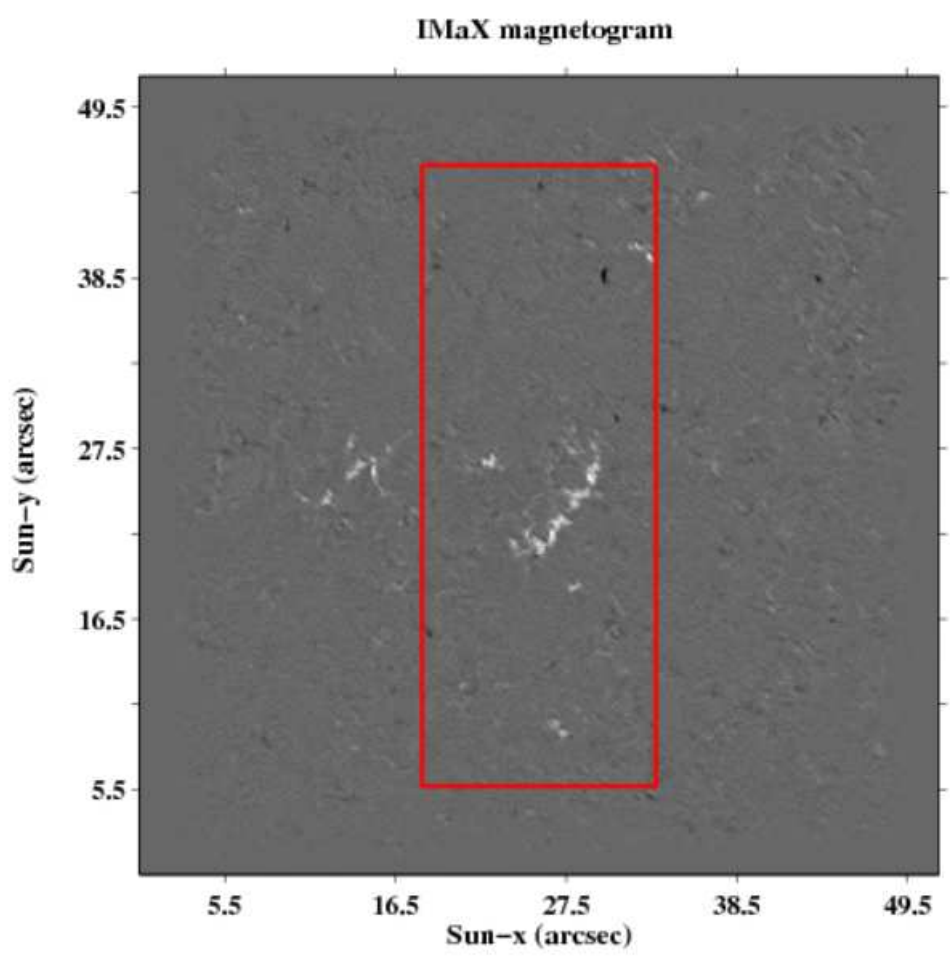

BPs in SuFI Data

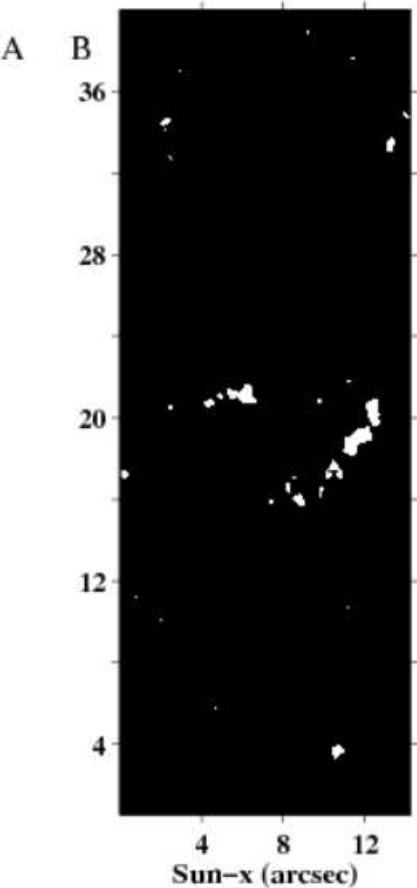

Figure 13. IMaX magnetogram (A) recorded on 9 June 2009 (14:34:18 UT), identified BPs in a SuFI image recorded at the same time (B). The red box in the magnetogram shows approximately the same region as covered by the SuFI image. Individual and network BPs are observable in both images. The comparison between IMaX megnetogram with the SuFI image, which includes BPs identified with our method, clearly yields compatible results. 
SOLA2282R4.tex; 16/06/2022; $7: 52 ;$ p.24 